\title{
TEG-seq: an ion torrent-adapted NGS workflow for in cellulo mapping of CRISPR specificity
}

\author{
Pei-Zhong Tang*,1, Bo Ding ${ }^{1}$, Lansha Peng ${ }^{1}$, Vadim Mozhayskiy ${ }^{1}$, Jason Potter $^{1}$ \& \\ Jonathan D Chesnut ${ }^{1}$ \\ ${ }^{1}$ Thermo Fisher Scientific Inc., R\&D Synthetic Biology, 5781 Van Allen Way, Carlsbad, CA 92008, USA
}

BioTechniques 65: 259-267 (November 2018) 10.2144/btn-2018-0105

Keywords: CRISPR/cas9 • gene editing • GUIDE-seq • off-target • TEG-seq

GUIDE-seq was developed to detect CRISPR/Cas9 off-target. However, as originally reported, it was associated with a high level of nonspecific amplification. In an attempt to improve it, we developed target-enriched GUIDE-seq (TEG-seq). The sensitivity level reached 0.1-10 reads-per-million depending on the NGS platform used, which was equivalent to 0.0002-1\% measured by Targeted Amplicon-seq. Application of TEGseq was demonstrated for the evaluation of various Cas9/gRNA configurations, which suggests delivery of Cas9/gRNA ribonucleoprotein results in significantly fewer off-targets than Cas9/gRNA plasmid. TEG-seq was also applied to $22 \mathrm{gRNAs}$ with relatively high in silico ranking score that targeted the biological relevant SNPs. The result indicated the initial selection of gRNAs with high score is important, although it cannot exclude the possibility of off-target.

The evolution of genome editing technologies holds promise for the concept of directly correcting mutations or disrupting abnormal genes in order to treat diseases, particularly inherited genetic disorders. Current databases indicate 135,588 variant-disease associations (VDAs), between 83,002 SNPs and 9169 diseases and phenotypes [1,2] in over 3874 genes [3]. These SNPs/mutations are potentially correctable or disruptable using genome editing tools such as CRISPR-Cas9 [4-7], TALEN [8-11] and ZFN [12-14]. As they are known to induce off-target mutations at sites with homology to the target sites, gene and cell therapeutic applications of these nucleases will require a comprehensive knowledge of their off-target effects to minimize the risk of deleterious outcomes.

Many strategies have been explored to improve the specificity of targeted nucleases. Modifications to the Fokl dimerization domain increased the specificity of ZFNs and TALENs by requiring formation of obligate heterodimers to cleave the target DNA in a prescribed orientation and spacing $[15,16]$. The inactivation of Cas 9 nuclease domains to create Cas9 nickases or dCas9-Fokl fusions has also increased specificity by requiring two gRNA/Cas9 complexes [17-20]. Likewise, reducing the gRNA length from 20 to 17 nucleotides has been shown to increase specificity [21]. Recently, structure-guided protein engineering [22-24] and phageassisted continuous evolution [25] have been used to develop novel Cas9 variants that show reduced off-target cleavage. These improvements have reduced initial concerns over the specificity of CRISPR/ Cas9 nucleases. However, regardless of the nuclease technology, it is difficult to determine the full spectrum of off-target cleavage events in a complex genome under various experimental conditions. That said, an efficient, unbiased and reliable genomewide off-target detection method is crucial for the application of genome editing-based gene and cell therapy as well as for benchmarking the fidelity evaluation of different gene editing tools.

Various methods have been developed to identify off-target, nuclease-cleaved sites. These methods rely on the double-strand breaks (DSBs) caused by nucleases that can be marked by a DNA tag either through in vitro ligation or in cellulo tag integration. The marked DSB site is then amplified and sequenced using next-generation sequencing (NGS). Several in vitro methods including BLESS-seq [26], HTGTS [27] and CIRCLE-seq [28] have been developed for off-target detection. These methods, by nature of the fact that the genomic DNA substrate has been removed from a cellular context and stripped of all protein, tend to identify all possible on- and off-target cleavage sites for a particular gRNA. The data analysis can also be challenging due to the potentially high nonspecific sequence reads as a result of the $\mathrm{PCR}$ amplification used to enrich the marked DSB. Digenomeseq [29], another in vitro method that relies on whole-genome sequencing, may reduce the noise and false positive calls, but likely sacrifices the sensitivity necessary to faithfully identify off-target sites that are cleaved at low frequencies. In cellulo approaches, in which off-target cleavage events occur and are tagged in living cells, more closely simulate the cellular nuclease-based gene editing environment. Two methods, IDLV-seq [30] and GUIDE-seq [31] were developed with this goal. IDLV-seq relies

TEG-seq is a target-enriched GUIDE-seq using an ion-torrent next-generation sequencing platform for unbiased genome-wide CRISPR off-target detection. It reduces nonspecific amplification, and therefore increases its sensitivity for off-target detection. 
on the integration of a linear viral DNA to the DSB site. However, the viral DNA is not chemically protected, which could lead to partial degradation and low efficiency of tag integration into DSBs and low sensitivity of detection. GUIDE-seq uses phosphorothioate-modified double strand DNA oligos (dsOND) as tags to reduce degradation and improve tag integration efficiency at DSBs in living cells. GUIDE-seq also requires an exponential PCR amplification step to enrich the marked DSB sites that may cause higher nonspecific noise and potential falsepositive calls.

We report here a modification of the GUIDE-seq method, termed targetenriched GUIDE-seq (TEG-seq), in which 5' phosphate primers are used for PCR amplification and differentially mark the amplicon containing the DSB site from nonspecifically amplified products. This is followed by preferential magnetic bead enrichment of marked DSB amplicons. These improvements significantly reduce nonspecific amplification and improve sensitivity of DSB detection. We also developed a 96-well format workflow that enables the study of multiple samples in parallel. The sensitivity of this method reached the level of Targeted Amplicon-seq, which has been widely used to validate the known and predicted gRNA off-target sites through direct amplification and NGS [28,31].

\section{Materials \& methods}

Oligonucleotides, double strand DNA Tag \& adaptors

The oligonucleotide sequences for TEG-seq and Targeted Amplicon-seq are listed in Supplementary Table 5. The double strand DNA (dsTag) and adaptors were annealed in the reaction containing $1 \times$ TE buffer, $100 \mathrm{mM} \mathrm{NaCl}$ and $50 \mu \mathrm{M}$ of top and bottom oligos, at $75^{\circ} \mathrm{C}$ for $5 \mathrm{~min}$ and gradually cooled down to room temperature in 20 to $30 \mathrm{~min}$.

gRNA preparation

The gRNAs were synthesized using in vitro transcription as described in a previous publication [32]. Briefly, the $80 \mathrm{bp} c r / t r a c r R N A$ constant region was $\mathrm{PCR}$ amplified from the GeneArt ${ }^{\circledR}$ CRISPR Nuclease Vector (1 ng) using the Constant Forward and Universal Reverse oligos $(10 \mu \mathrm{M})$. The $80 \mathrm{bp} \mathrm{cr}$ / tracrRNA PCR products $(0.15 \mu \mathrm{M})$ were equally mixed with universal forward and reverse oligos $(10 \mu \mathrm{M})$ as well as target- specific forward and reverse oligos $(0.3 \mu \mathrm{M})$. The in vitro transcription of gRNA was carried out using the TranscriptAid T7 High Yield Transcription Kit (ThermoFisher Scientific, MA, USA) following the manufacturer's protocol.

\section{SpCas9 expression}

For the plasmid format, the SpCas9 and gRNA were cloned in the GeneArt CRISPR Nuclease Vector (ThermoFisher Scientific) following the manufacturer's protocol. The recombinant wild type SpCas9 (Platinum $^{\text {TM }}$ Cas9) were purchased from ThermoFisher Scientific).

\section{TEG-seq using the ion}

torrent platform

See the protocol provided in the Supplementary data.

Ion-torrent NGS \& data analysis for TEG-seq \& Targeted Amplicon-seq Ion-torrent platform was used for all sequencing. $\mathrm{PGM}^{\mathrm{TM}}$ with 318-chip or Proton with PI-chip was used for TEG-seq to obtain at least one million reads per sample. Proton with PI-chip or S5 with 540-chip were used for Target Ampliconseq to obtain at least 10,000 reads per sample. Reads were first aligned to the human genome reference, hg19. Mapped reads were further processed through in-house developed plug-in software, named Motif-Search, which performs the following: sorts barcodes for different samples, stacks and counts reads for all amplicons, and scans the genome and identifies the sequence and genome position for potential off-targets based on the guide and PAM sequence. The candidates for potential CRISPR/Cas9-induced DSB sites were compared with the control sample lacking CRISPR/Cas9 treatment (dsTag only) to determine if the candidates were related to CRISPR/Cas9-induced DSB sites. To compare different samples from various experiments and different NGS runs, reads from all samples were normalized using reads per million (RPM of mapped read).

NGS reads from the Targeted Amplicon-seq experiments were aligned to the corresponding reference PCR sequence. The mapped reads were further processed using the in-house developed plugin, named 'CELFT' (cut efficiency for low frequency target) to visualize the cleavage that contains 'large indel' and/or dsTag integration and calculated percentage of cleavage events. To minimize the false positive for the Targeted Amplicon-seq validation, caused by NGS error, especially the area with homopolymer in cleavage loci, only the large indel for having at least three or more bases was counted as a positive.

\section{Results \& discussion}

The TEG-seq workflow

GUIDE-seq [31] and other genome-wide off-target detection methods [26-28,30] have been developed to provide relatively unbiased mapping of on- and off-target cleavage events. These technologies depend on the exponential amplification of tagged segments of the genome followed by sequencing and identification of those amplicons. While this technology has proven to be an advancement to the field, it can be associated with high background reads and a technically challenging workflow. Using a similar dsTag as previously reported [31], we tested the GUIDE-seq workflow using ion-torrent NGS (Ion-GUIDE-seq; Supplementary Figure 1 and 'Materials \& methods'). The sequencing data showed that only a small portion of amplicons contained the desired dsTag sequence with the majority of the high-read hits not related to CRISPR/Cas9 editing (data not shown). It was hypothesized that the majority of PCR products amplified using the dsTag-specific primers ( $F$ or $\mathrm{R}$ ) paired with the adaptor primer (P1) were actually the P1/P1 product (Supplementary Figure 1). This was confirmed by using the P1 primer alone under various conditions for multiple samples (Supplementary Figure 2). This over-representation of the P1/P1 product likely impaired the sensitivity of Ion-GUIDE-seq for genomewide off-target detection.

To reduce the undesired $\mathrm{P} 1 / \mathrm{P} 1$ product amplified in Ion-GUIDE-seq and enrich for the targeted DSB amplicons for NGS, we developed the TEG-seq sample preparation procedure. As shown in Figure 1, we used 5'-phosphorylated primers paired with the P1 primers for the secondround PCR amplification. This resulted in a 5'-phosphate on the products (5P-F2) $\mathrm{P} 1$ and 5P-R2/P1) but not on the P1/P1 product. In the next step, a nonphosphorylated barcoded adaptor (BC-A), containing the ion sequencing primer $A$ was specifically ligated to the phosphorylated product 
but not the P1/P1 product, which lacks the $5^{\prime}$ phosphate required to ligate the nonphosphorylated barcode adaptor. A third round of PCR was then performed using P1 and 'A-tail' primers to further enrich the P1-A product. The BC-A-tail primer contains an internal spacer that stops the polymerase-mediated nucleotide extension and leaves a singlestrand tail at the end of the PCR product. Finally, the dsTag-specific A-P1 product was further enriched from the remaining P1/P1 nonspecific product using a biotinylated oligo complimentary to the tail sequence and captured by streptavidin (SA) magnetic bead selection. The barcoded A-P1 product was then sequenced. By selectively modifying target PCR amplicons using 5'phosophorylated primers followed by bead enrichment for the targeted fragments, TEG-seq reduces nonspecific amplification and therefore likely increases sensitivity. We also used barcoding to multiplex the workflow to improve robustness and throughput.

\section{Validation of TEG-seq accuracy} using Targeted Amplicon-seq

To evaluate the sensitivity level of TEG-seq, we compared it with Targeted Amplicon re-sequencing using NGS (Targeted Amplicon-seq), which we believe is currently the most sensitive method to detect nuclease-induced editing at predicted chromosomal locations. The two gRNAs using targeted loci HEK4 and VEG1 (previously identified to have a high and medium number of off-target sites [31] with poor in silico ranking score (Supplementary Table 1) were chosen. We detected over 300 and 30 off-target candidates for HEK4 and VEG1 respectively from the TEG-seq experiments and confirmed 253 positive for HEK4 and 28 positive for VEG1 by Targeted Amplico-seq. The rarest off-target event detected by TEG-seq was approximately 1 RPM for HEK4 from 5 million and 4 RPM for VEG1 from 2.4 million total reads (Figure 2A and 2B, Supplementary Table 2). The rarest off-target detected by Targeted Ampliconseq was $0.0004 \%$ for HEK4 and $0.0002 \%$ for VEG1. There was a reasonable correlation between TEG-seq and Targeted Amplicon-seq for both HEK4 and VEG1 with the correlation factor $R$ value at 0.9070 and 0.9329 respectively.

Taken together, these data suggest that using NGS platforms such as PGM that produce several million total reads in a single run, TEG-seq is capable of detecting off-target events at levels as low as 1-10 RPM, which corresponds to the cleavage rate as low as $0.001-1 \%$ as detected by Targeted Amplicon-seq (Figure 2). The combination of TEG-seq for profiling off-targets followed by validation using Targeted Amplicon-seq could be a good approach to identify appropriately edited cell lines and to validate cells destined for use in cell and gene therapy.
Off-target detection sensitivity under various sequencing depths

The sensitivity of TEG-seq and GUIDE-seq detection is directly proportional to the sequencing depth. The data from our lab and others [28,31] were generated using either lon-Torrent PGM or Illumina MiSeq, which yield approximately $1-5$ million reads per run. The lower limit of detection using the PGM was approximately 1-10 RPM. It is likely that rarer off-target events could be detected by increasing the depth of

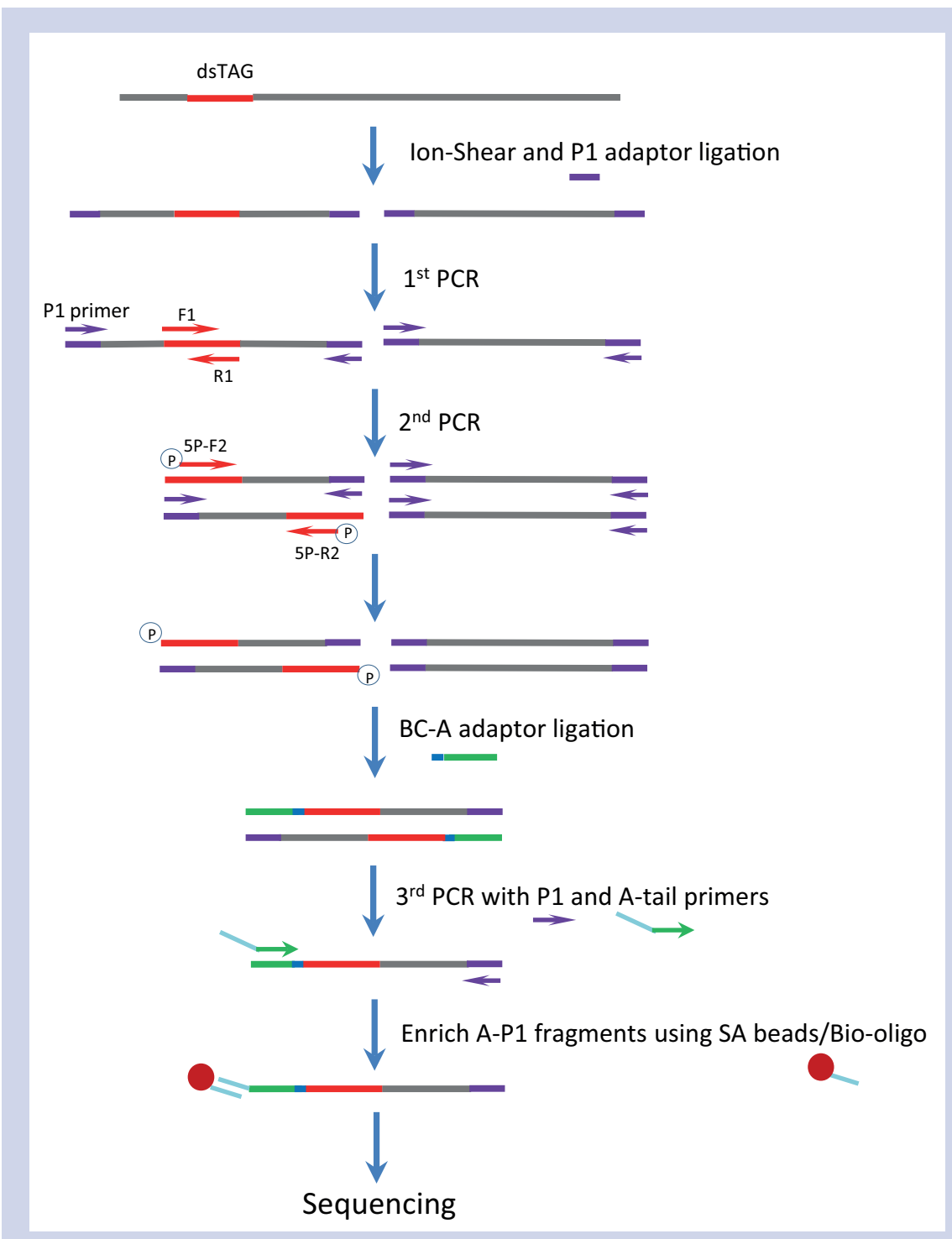

Figure 1. Diagram of ion-TEG-seq workflow. A dsTag was co-transfected with Cas9/gRNA ribonucleoprotein or plasmid that expresses Cas9 and gRNA. The genomic DNA was extracted and fragmented to 200-600 bp using enzyme-based ion-shear. The P1 adaptor was ligated to the fragmented DNA sample. The first PCR was performed in separated tubes using P1/F1 for the forward and P1/R1 for the reverse PCR. In the second (nested) PCR, 5' phosphorylated primers (5P-F2 or 5P-R2) were used that generated PCR products containing a 5' phosphate only in the F2/P1 or R2/P1 products, but not P1/ P1 product. A nonphosphorylated barcode adaptor (BC-A) was specifically ligated to the F2/P1 or R2/ P1 products, but not to the P1/P1 product. A third PCR was performed using P1/A-tail primer followed by a bead enrichment via a biotinylated capture oligo that was complementary to the A-tail primer. The enriched amplicons were then applied to next-generation sequencing.

dsTag: Double strand DNA tag; SA: Streptavidin. 

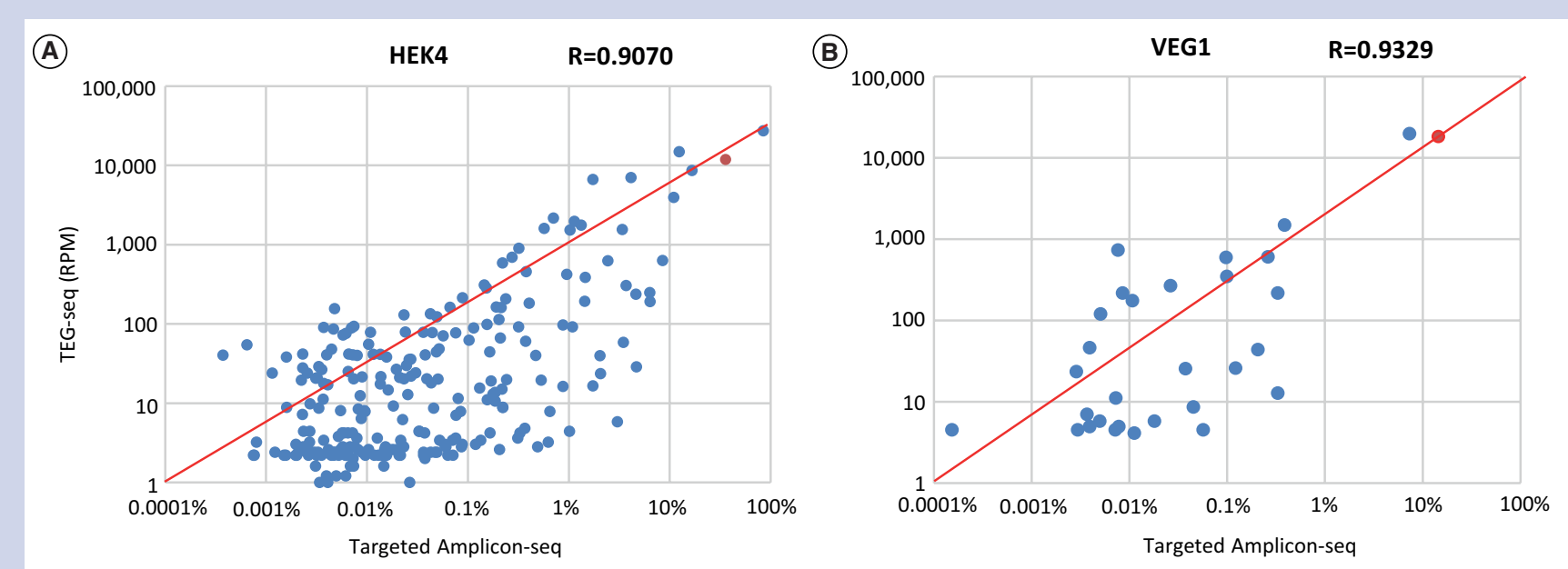

Figure 2. Comparison of off-target detection level between TEG-seq and Targeted Amplicon-seq. (A) HEK4 off-targets detected by TEG-seq is plotted in RPM against the percentage of cleavage detected by Targeted Amplicon-seq. The correlation factor $\mathrm{R}$ value is indicated on the upper right side. The on-target activity is indicated by red color. (B) VEG1 off-targets detected by TEG-seq in RPM is plotted to the percentage of cleavage detected by Targeted Amplicon-seq. The correlation factor $\mathrm{R}$ value is indicated on the upper right side. The on-target activity is indicated by red color. RPM: Reads-per-million.

sequencing. To verify this, we used the Ion Proton and re-sequenced the HEK4 sample that was delivered with wild-type
SpCas9 RNP. The Proton generated 42 million mapped reads, which is approximately 23-fold higher than the number of reads (1.8 million) from PGM. With this increased depth, we detected 32 additional off-target events. The read number per

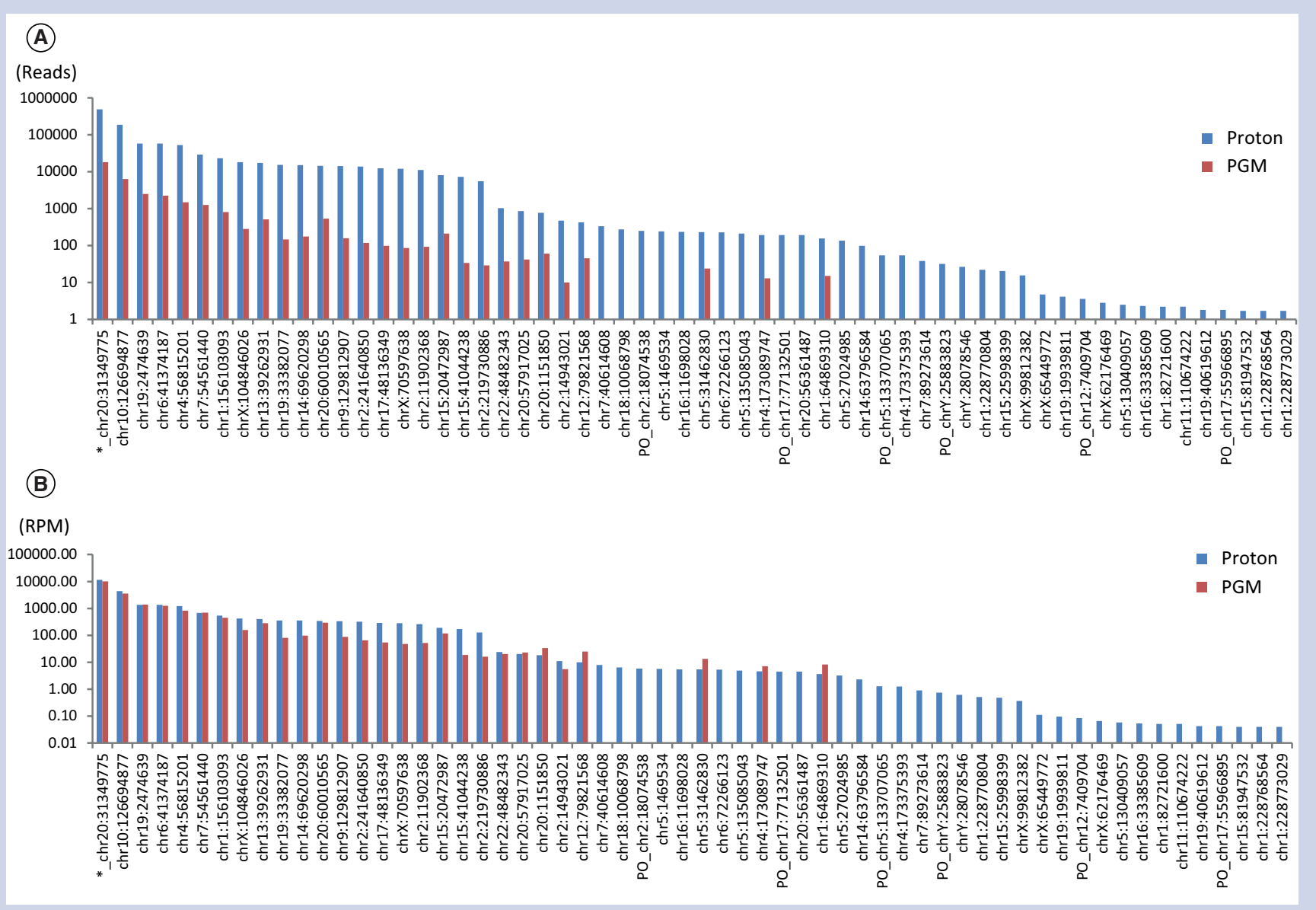

Figure 3. Off-target detection of HEK4 gRNA-directed cleavage at different sequencing depths. (A) Read count of HEK4 on- and off-targets. (B) RPM of HEK4 on- and off-targets. Asterisks indicate on-target events.

RPM: Reads-per-million. 


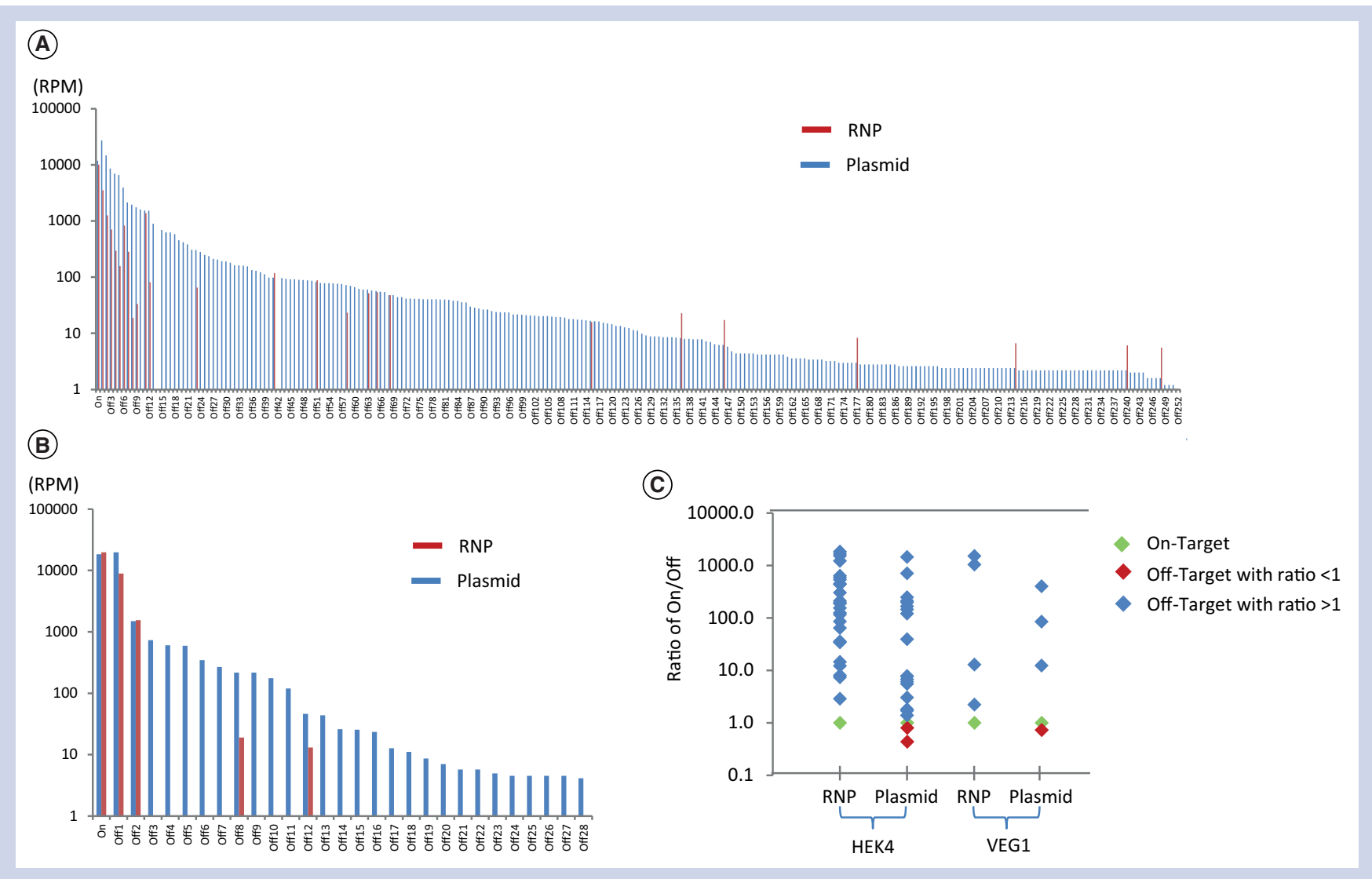

Figure 4. Off-target mapping using Cas9/gRNA plasmid and RNP delivery formats. (A) On- and off-target events detected using plasmid or RNP Cas9 with HEK4 site gRNA. (B) On- and off-target events detected using plasmid or RNP Cas9 with VEG1 site gRNAs. (C) Pairwise comparison of the on-/off-target ratio for those targets that were positive from both plasmid and RNP delivering formats.

RNP: Ribonucleoprotein; RPM: Reads-per-million.

target from the Proton is approximately a one order of magnitude increase over PGM (Figure 3A). However, their off-target profiles were similar after normalizing the read number to RPM with the additional, more rare 32 off-targets (Figure 3B). All 32 additional off-target events detected by Proton were below 8 RPM and ranged down to 0.04 RPM (Supplementary Table 3). 26 of the 32 additional off-target events detected by the Proton were also detected by PGM when the plasmid format was used. However, six appeared novel and not previously detected in this or other studies published [28,31]. These data indicate that with the throughput of 1-5 million reads generated by the PGM, off-target loci can be detected at the level of 1-10 RPM, which is equivalent to $0.001-1 \%$ from Targeted Amplicon-seq. Deeper sequencing platform such as Ion Proton or ion S5 would be recommended to detect lower than 1-10 RPM off-targets.

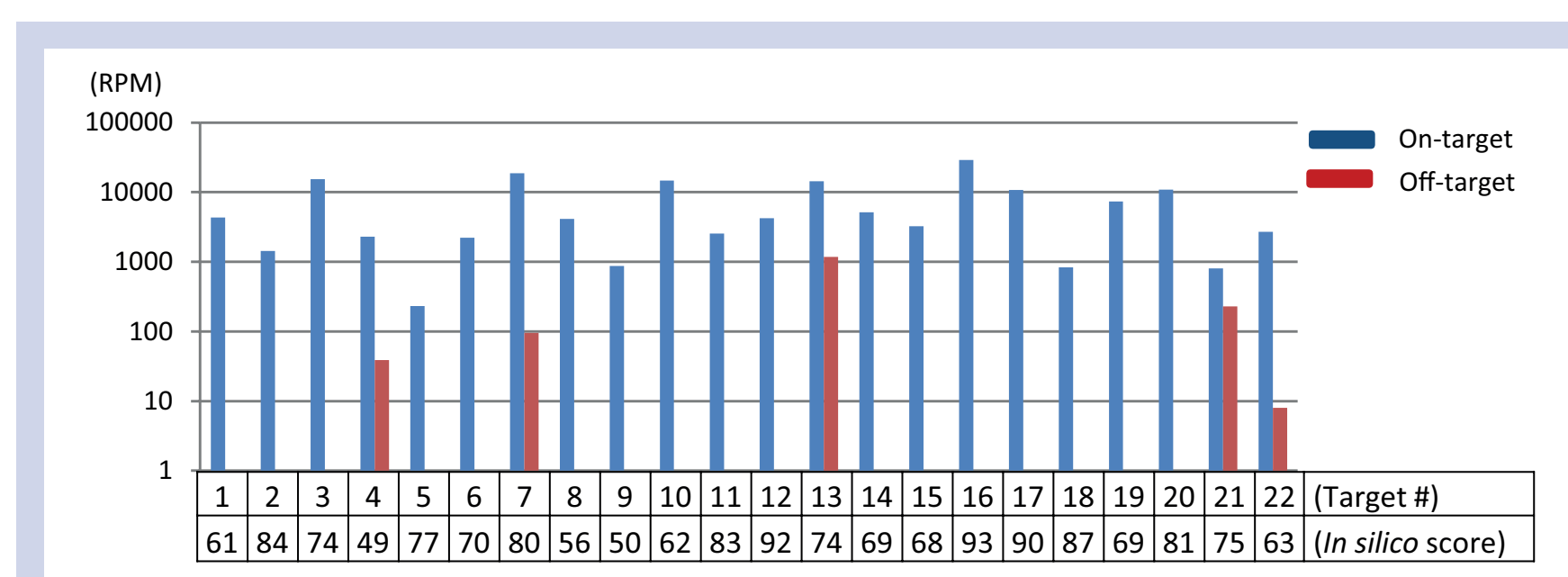

Figure 5. On- and off-targets detected from 22 gRNAs targeting biological relevant SNPs. 22 gRNAs with in silico ranking score from MIT CRISPR design tool targeting eight disease-associated SNPs (Supplementary Table 4). Their on- and off-targets were detected by TEG-seq and measured by PRM. 
Table 1. The detailed result of five gRNAs detected with a single off-target.

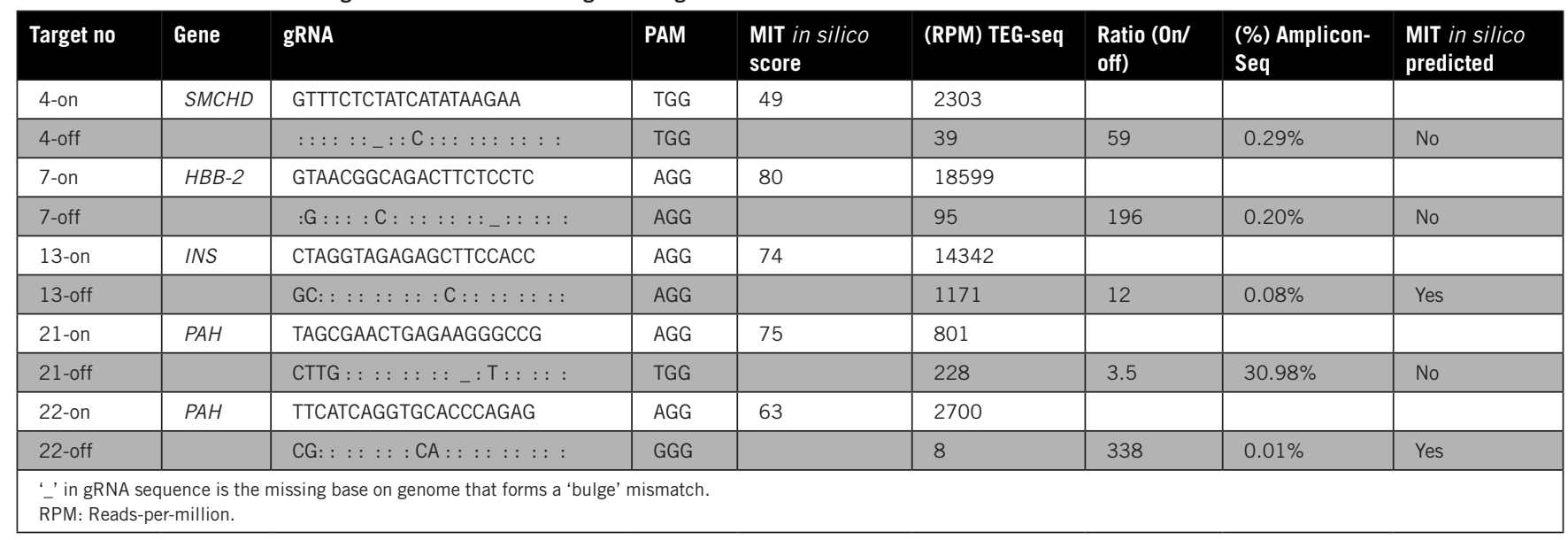

Comparison of genome-wide off-target cleavage using plasmid \& RNP CRISPR/Cas9 formats

In comparison with Cas9 delivered to the cell via plasmid DNA, direct transfection of Cas9 protein/gRNA ribonucleoprotein complexes (RNPs) provides a transient burst of activity with no opportunity for direct integration and persistent cleavage activity [32,33]. The Cas9/gRNA RNP is quickly turned over by the cell, which likely lowers the cellular concentration and thus the opportunity for off-target cleavage. CRISPR/Cas9 RNP delivery has grown in popularity but validation of genome-wide off-target effects among wild-type and various 'high fidelity' Cas9 as RNPs has only been reported recently [28]. This type of specificity evaluation benchmarking of various RNPs will be important particularly for therapeutic applications where the Cas9 plasmid format is not optimal. In an effort to more clearly define the differences in specificity between Cas9 plasmid and RNP delivery, we applied TEG-seq to HEK4 and VEG1 gRNAs and found that RNP delivery yielded approximately nineand six-fold fewer detectable off-target events (25 vs 252 and 4 vs 27) respectively as compared with Cas9 plasmid delivery (Figure 4A \& B). In general we found significantly lower off-target RPM with the RNP format than the plasmid format while their RPM of on-targets were similar. More importantly, the ratio of on-/ off-target was considerably higher with RNPs than with the plasmid format in a pairwise comparison to those positive off-targets from both formats (Figure 4C). In contrast to plasmid delivery, we did not detect any off-target events from the RNP delivery format that had higher cleavage activity than the on-target (the ratio of on-/ off-target $<1$ ). Although the results showed marked improvement with RNP delivery, a subset of significantly active off-target sites remain.

These results support the idea that using Cas9 as RNP reduces the number of detectable off-target events and improves overall specificity over plasmid DNA as measured by the ratio of on-target to off-target events.

This is important when considering cell line development where a tenfold difference in on-target activity to the next highest off-target event represents an approximate one in ten chance of isolating a cell line with both events occurring in the same genome. Conversely, for therapeutic applications of Cas9 RNPS in nondividing primary cells, complete elimination of off-target events would be desirable as clonal isolation is generally not achievable prior to application of cells to the patient.

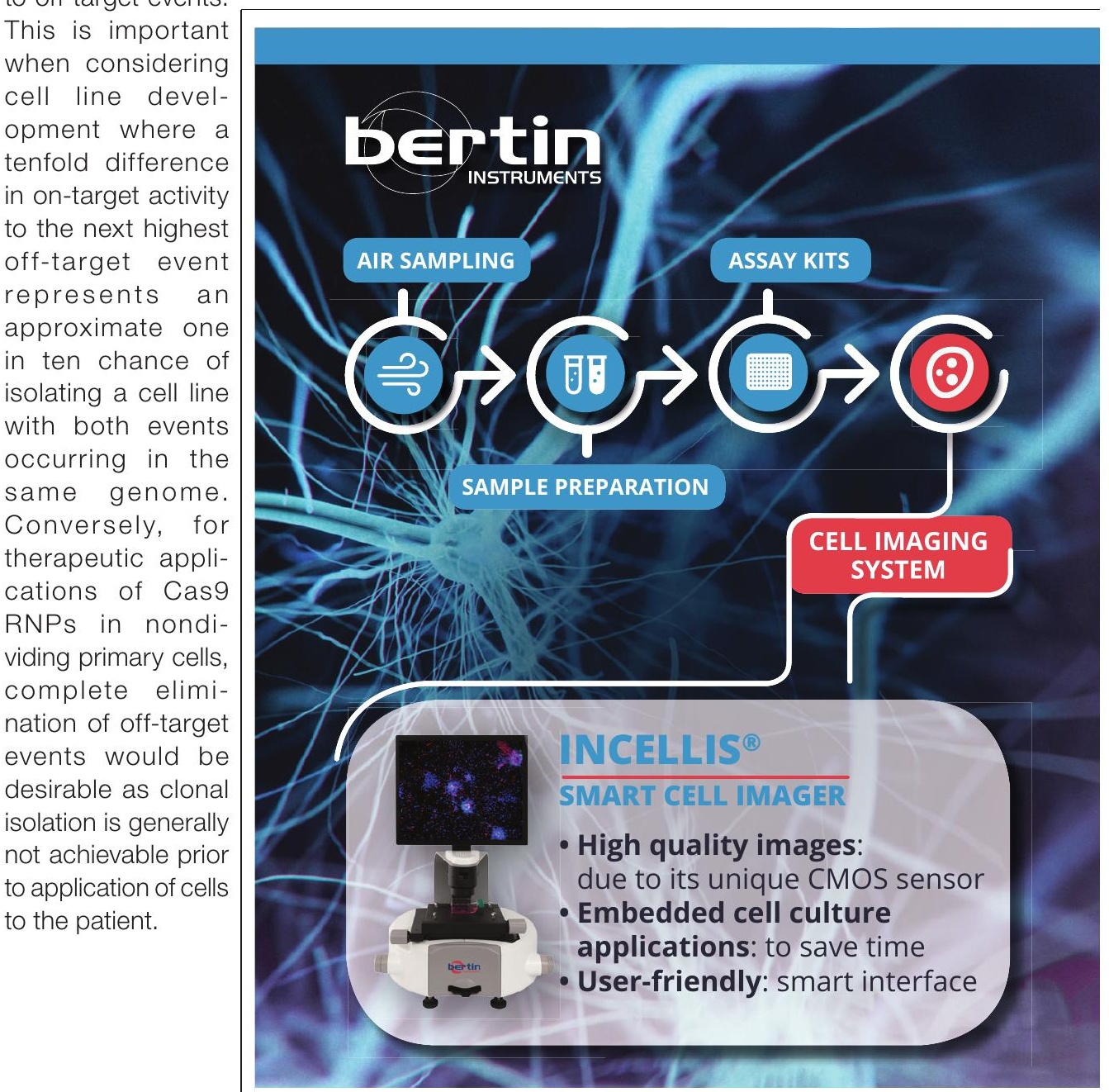

Genome wide off-targets on 22 gRNAs with relative high ranking score designed from in silico tool Significant effort has been made to create a rule set to enable better in silico prediction of CRISPR specificity $[34,35]$. However, there remains a relatively large discrepancy between off-targets detected by nonbiased approaches with those predicted using the current in silico design 
tools [28,31]. It's worth noting that the gRNAs (HEK4 and VEG1) evaluated in this study and those in previous work $[28,31]$ had poor in silico ranking score (Supplementary Table 1). They were chosen specifically due to their propensity for high off-target cleavage. We felt it pertinent to apply the nonbiased approach to evaluate the gRNAs with relative high in silico ranking score to simulate the application scenario of gene correction. We chose 22 gRNAs targeting the SNPs that cause eight genetic disorders (Supplementary Table 4) using the wild-type SpCas9 RNP delivery format. Surprisingly, only five $(23 \%)$ of 22 resulted in detectable single off-target cleavage events by TEG-seq and confirmed by Targeted Amplicon-seq (Figure 5 and Table 1). The off-target RPMs from TEG-seq were at least one order of magnitude lower compared with their corresponding on-target except the \#21. The percentages of off-target cleavage from Amplicon-seq were all <0.29\% except the \#21. The in silico ranking score seem not well-correlated to the off-target result. For example, three targets (\#7, \#13 and \#21) with the detectable off-targets have a relatively high ranking score. Of the five targets with detected off-target, only two were predicted by the design tool; three were not predicted, in which a 'bulge' mismatch was contained. The result implies that the initial selection of gRNA with high in silico ranking score is important although it cannot exclude the off-target. The probabilityof having the off-target is low if using Cas9/gRNA RNP delivery format with a high in silico score gRNA. To minimize detectable off-target, it is wise to design and test two to three gRNAs for each target.

This study reports an adaptation of the originally reported GUIDE-seq method to the Ion Torrent family of NGS instruments. The new protocol reduces nonspecific sequencing reads by enriching for relevant amplicons prior to sequencing while also enabling multiplexing of experiments through barcoding. TEG-seq represents an improved tool for validating CRISPR nuclease specificity in a cellular context. The nonbiased detection methods such as TEG-seq could be used for initial specificity screening of gRNAs followed by validation of the final edited cell clones using deep Targeted Amplicon-seq to identify appropriately edited cell lines and to validate cells destined for use in cell and gene therapy.

\section{Author contributions}

Pei-Zhong Tang developed the method, designed and performed most of bench work, analyzed and generated data and drafted the manuscript. Lansha Peng performed some bench work. Bo Ding and Vadim Mozhayskiy developed bioinformatic tools. Jason Potter and Jonathan D Chesnut edited the manuscript.

\section{Acknowledgments}

The authors would like to thank John Bishop and Steven J Roman for the initial technical advises and discussion.

\section{Open access}

This work is licensed under the Attribution-NonCommercial-NoDerivatives 4.0 Unported License. To view a copy of this license, visit http://creativecommons.org/ licenses/by-nc-nd/4.0/

\section{Competing \& financial interests disclosure}

The authors have no relevant affiliations or financial involvement with any organization or entity with a financial interest in or financial conflict with the subject matter or materials discussed in the manuscript. This includes employment, consultancies, honoraria, stock ownership or options, expert testimony, grants or patents received or pending, or royalties.

No writing assistance was utilized in the production of this manuscript.

\section{Supplementary data}

To view the supplementary data that accompany this paper please visit the journal website at: www.future-science. com/doi/full/10.2144/btn-2018-0105

\section{References}

1. DisGeNET. www.disgenet.org/web/DisGeNET/ menu;jsessionid=4aqu6smfdc4c13gfeo1lsnaxl

2. Clinvar. www.ncbi.nlm.nih.gov/clinvar

3. OMIM Gene Map Statistics. www.omim.org/ statistics/geneMap

4. Mali P, Yang L, Esvelt KM et al. RNA-guided human genome engineering via Cas9. Science 339(6121), 823-826 (2013).
5. Cong L, Ran A, Cox D et al. Multiplex genome engineering using CRISPR/Cas systems. Science 339, 819-823 (2013).

6. Kim S, Kim D, Cho SW, Kim J, Kim JS. Highly efficient RNA-guided genome editing in human cells via delivery of purified Cas 9 ribonucleoproteins. Genome Res. 24(6), 1012-1019 (2014).

7. Schumann K, Lin S, Boyer E et al. Generation of knock-in primary human T cells using Cas 9 ribonucleoproteins. Proc. Natl Acad. Sci. USA 112(33), 10437-10442 (2015).

8. Kim Y, Kweon J, Kim A et al. A library of TAL effector nucleases spanning the human genome. Nat. Biotechnol. 31(3), 251-258 (2013).

9. Kim YK, Wee G, Park J et al. TALEN-based knockout library for human microRNAs. Nat. Struct. Mol. Biol. 20(12), 1458-1464 (2013).

10. Miller JC, Tan S, Qiao G et al. A TALE nuclease architecture for efficient genome editing. Nat. Biotechnol. 29(2), 143-148 (2011).

11. Mussolino C, Morbitzer R, Lütge F, Dannemann $\mathrm{N}$, Lahaye T, Cathomen T. A novel TALE nuclease scaffold enables high genome editing activity in combination with low toxicity. Nucleic Acids Res. 39(21), 9283-9293 (2011).

12. Bibikova M, Beumer K, Trautman JK, Carroll D. Enhancing gene targeting with designed zinc finger nucleases. Science 300(5620), 764 (2013).

13. Kim HJ, Lee HJ, Kim H, Cho SW, Kim JS. Targeted genome editing in human cells with zinc finger nucleases constructed via modular assembly. Genome Res. 19, 1279-1288 (2009).

14. Kim JS, Lee HJ, Carroll D. Genome editing with modularly assembled zinc-finger nucleases. Nat. Methods 7, 91-92 (2010)

15. Miller JC, Holmes MC, Wang J et al. An improved zinc-finger nuclease architecture for highly specific genome editing. Nat. Biotechnol. 25(7), 778-785 (2007).

16. Guo J, Gaj T, Barbas CF III. Directed evolution of an enhanced and highly efficient Fokl cleavage domain for zinc finger nucleases. J. Mol. Biol. 400(1), 96-107 (2010).

17. Ran FA, Hsu PD, Lin CY et al. Double nicking by RNA-guided CRISPR Cas 9 for enhanced genome editing specificity. Cell 154(6), 13801389 (2013).

18. Mali P, Aach J, Stranges PB et al. CAS9 transcriptional activators for target specificity screening and paired nickases for cooperative genome engineering. Nat. Biotechnol. 31(9), 833-838 (2013).

19. Tsai SQ, Wyvekens N, Khayter C et al. Dimeric CRISPR RNA-guided Fokl nucleases for highly specific genome editing. Nat. Biotechnol. 32, 569-576 (2014).

20. Guilinger JP, Thompson DB, Liu DR. Fusion of catalytically inactive Cas9 to Fokl nuclease improves the specificity of genome modification. Nat. Biotechnol. 32, 577-582 (2014).

21. Fu Y, Sander JD, Reyon D, Cascio VM, Joung JK. Improving CRISPR-Cas nuclease specificity using truncated guide RNAs. Nat. Biotechnol. 32(6), 279-284 (2014).

22. Slaymaker IM, Gao L, Zetsche B, Scott DA, Yan WX, Zhang F. Rationally engineered Cas9 nucleases with improved specificity. Science 351, 84-88 (2016).

23. Kleinstiver BP, Pattanayak V, Prew MS et al. High-fidelity CRISPR-Cas9 nucleases with no detectable genome-wide off-target effects. Nature 529(7587), 490-495 (2016). 
24. Chen JS, Dagdas YS, Kleinstiver BP et al. Enhanced proofreading governs CRISPR-Cas9 targeting accuracy. Nature 550(7676), 407-410 (2017).

25. Hu JH, Miller SM, Geurts MH et al. Evolved Cas9 variants with broad PAM compatibility and high DNA specificity. Nature 556(7699), 57-63 (2018).

26. Crosetto N, Mitra A, Silva MJ et al. Nucleotideresolution DNA double-strand break mapping by next-generation sequencing. Nat. Methods 10(4), 361-368 (2013)

27. Frock RL, Hu J, Meyers RM et al. Genomewide detection of DNA double-stranded breaks induced by engineered nucleases. Nat. Biotechnol. 33(2), 179-187 (2015).

28. Tsai SQ, Nguyen NT, Malagon-Lopez J, Topkar VV, Aryee MJ, Joung JK. CIRCLE-seq: a highly sensitive in vitro screen for genomewide CRISPR-Cas9 nuclease off-targets. Nat. Methods 14(6), 607-614 (2017).
29. Kim D, Bae S, Park J et al. Digenome-seq: genome-wide profiling of CRISPR-Cas9 off-target effects in human cells. Nat Methods 12(3), 237-243 (2015).

30. Wang X, Wang Y, Wu Xet al. Unbiased detection of off-target cleavage by CRISPR-Cas 9 and TANENs using integrase-defective lentiviral vectors. Nat. Biotechnol. 33(2), 175-179 (2015).

31. Tsai SQ, Zheng Z, Nguyen NT et al. GUIDE-seq enables genome-wide profiling of off-target cleavage by CRISPR-Cas nucleases. Nat. Biotechnol. 33(2), 187-197 (2015).

32. Liang $X$, Potter J, Kumar $S$ et al. Rapid and highly efficient mammalian cell engineering via Cas9 protein transfection. J. Biotech. 208, 4453 (2015).

33. Kim S, Kim D, Cho SW, Kim J, Kim JS. Highly efficient RNA-guided genome editing in human cells via delivery of purified Cas9 ribonucleoproteins. Genome Res. 24(6), 1012-1019 (2014).

34. Doench JG, Hartenian E, Graham DB et al. Rational design of highly active sgRNAs for
CRISPR-Cas9-mediated gene inactivation. Nat. Biotechnol. 32(12), 1262-1267 (2014).

35. Doench JG, Fusi N, Sullender M et al. Optimized sgRNA design to maximize activity and minimize off-target effects of CRISPR-Cas9. Nat. Biotechnol. 34(2), 184-191 (2016).

First draft submitted: 17 July 2018;

Accepted for publication: 7 August 2018

Address correspondence to: Pei-Zhong Tang; Thermo Fisher Scientific Inc., R\&D Synthetic Biology, 5781 Van Allen Way, Carlsbad, CA 92008, USA; E-mail: pei-zhong.tang@thermofisher.com

\section{To purchase reprints of this article contact:} s.cavana@future-science.com

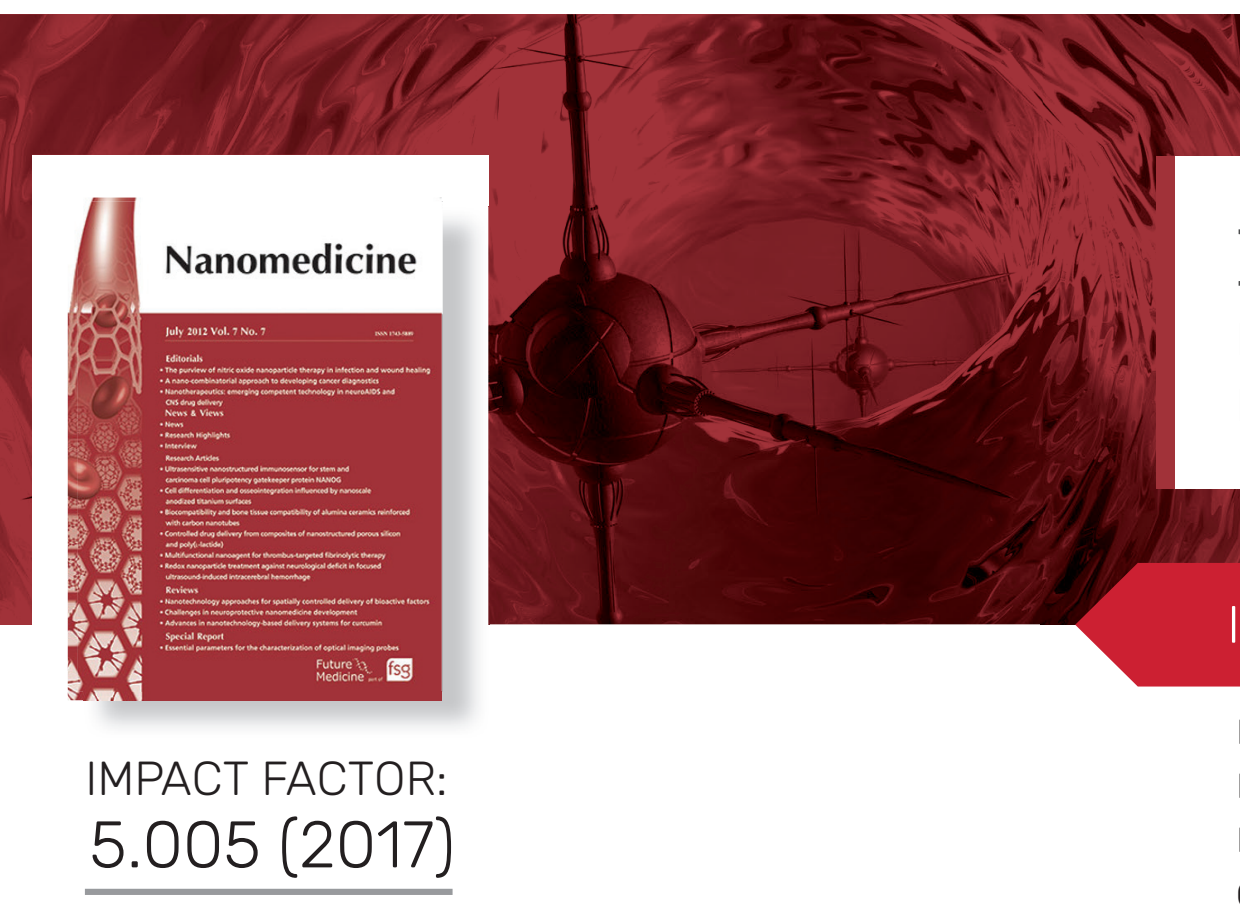

\section{Nanomedicine}

ISSN: 1743-5889

Frequency per year: 24

\section{INDEXING}

MEDLINE/Index Medicus, EMBASE/Excerpta

Medica, Chemical Abstracts, Science Citation Index Expanded ${ }^{\mathrm{TM}}\left(\mathrm{SciSearch}^{\circledR}\right)$ ), Biotechnology Citation Index ${ }^{\circledR}$, Current Contents ${ }^{\circledR} /$ Life Sciences, Journal Citation Reports/Science Edition ${ }^{\circledR}$, Inspec ${ }^{\circledR}$, Scopus

\section{www. futuremedicine.com}

To claim your free trial, contact us at: trials@futuremedicine.com quoting NNMBTN
Future $\because$. an imprint of Medicine 